\title{
Qualidade de vida dos graduandos em farmácia da Universidade Federal do Oeste do Pará
}

\author{
Quality of life of the graduating in pharmacy of the \\ Federal University of the West of Para
}

\author{
Luciana Fernandes Pastana Ramos', Juliana Érica Cirino Nascimento² \\ 'Instituto de Saúde Coletiva, Universidade Federal do Oeste do Pará, Santarém (PA), Brasil. \\ ${ }^{2}$ Universidade Federal do Oeste do Pará, Santarém (PA), Brasil.
}

Recebido: Ago. 14, 2017

Aceito: Nov. 21, 2017

\section{COMO CITAR ESTE ARTIGO}

Ramos LFP, Nascimento JEC. Qualidade de vida dos graduandos em farmácia da Universidade Federal do Oeste do Pará. Interdisciplinary Journal of Health Education 2017 Jan-Jul:2(1):54-60 http:// dx.doi.org/10.4322/ijhe.2016.030

\section{CORRESPONDÊNCIA}

Luciana Fernandes Pastana Ramos Instituto de Saúde Coletiva Universidade Federal do Oeste do Pará Avenida Mendonça Furtado, 2946 sala 224, Bairro Fátima, CEP 68040-470, Santarém (PA), Brasil

lucianafpramos@gmail.com.

\section{FONTE DE FINANCIAMENTO \\ Fundação Amazônia de Amparo a Estudos e Pesquisas do Pará (FAPESPA).}

\section{CONFLITO DE INTERESSE}

Os autores declararam não haver conflitos de interesse.

O estudo foi realizado na Universidade Federal do Oeste do Pará, Santarém (PA), Brasil.

Este trabalho foi apresentado no II Congresso Nacional Multidisciplinar da Saúde - CONMSAUDE. Realizado no Centro de Convencões de Goiânia, no período de 25 a 27 de maio de 2017 ao qual foi premiado com menção honrosa pela brilhante apresentação.

O trabalho foi aprovado pelo Comitê de Ética e Pesquisa com Seres Humanos da Universidade do Estado do Pará (UEPA) sob o parecer de ํㅜ 1.447 .073 e CAAE nำ53163815.0.0000.5168.

\section{RESUMO}

Objetivo: O objetivo deste estudo foi de investigar a qualidade de vida dos graduandos de Farmácia da Universidade Federal do Oeste do Pará (UFOPA). Métodos: Trata-se de um estudo prospectivo de corte transversal que foi realizado nos campus da UFOPA em Santarém. A pesquisa foi aprovada pelo Comitê de Ética em pesquisa da Universidade do Estado do Pará (CEP-UEPA). O tamanho total da amostra foi de 82 alunos, ambos os sexos, do 1ำ ao 4a ano de graduação do curso de Farmácia. Os instrumentos utilizados na obtenção dos dados foram: ficha de dados sóciodemográficos, instrumento de avaliação da qualidade de vida da Organização Mundial de Saúde abreviado (WHOQOL-BREF) e questionário Medical Outcomes Study 36-item Short-Form Health Survey (SF-36). Resultados: Os resultados sóciodemográficos caracterizaram a amostra com predomínio de gênero feminino ( $n=57 ; 69,5 \%)$; com idade entre 18 a 45 anos; solteiros $(n=74 ; 90,24 \%)$; religião católica ( $n=45 ; 54,88 \%$ ) e todos participantes relataram experiência de estágio. Conclusão: Os instrumentos WHOQOL-BREF e SF-36 apontam uma tendência de piores escores de QV no 1ํ e 2 ano curricular de Farmácia e os melhores escores de QV no 3ำ e 4 ano. Dessa forma, foi possível conhecer o perfil dos graduandos e analisar quais os domínios são mais prejudicados no percurso acadêmico.

PALAVRAS-CHAVE: Qualidade de vida. Estudantes de farmácia. Universidade.

\section{ABSTRACT}

Objective: The objective of this study was to investigate the quality of life of Pharmacy graduates of the Federal University of the West of Para (UFOPA). Methods: This is a prospective cross-sectional study that was carried out on the campus of UFOPA in Santarem. The research was approved by the Research Ethics Committee of the State University of Para (CEP-UEPA). The total sample size was 82 students, both sexes, from the 1 st to the 4th year of the pharmacy course. The instruments used to obtain the data were: sociodemographic data sheet, World Health Organization (WHOQOL-BREF) quality of life assessment instrument and Medical Outcomes Study 36-item Short-Form Health Survey (SF-36). Results: Sociodemographic data characterized the sample with a predominance of female gender ( $n=57,69.5 \%$ ); aged between 18 and 45 years; singles ( $n=74,90.24 \%$ ); Catholic religion ( $n=45 ; 54.88 \%$ ) and all participants reported internship experience. Conclusion: The WHOQOL-BREF and SF-36 instruments indicate a trend of worse QoL scores in the 1st and 2nd year Pharmacy curriculum and the best QoL scores in the 3rd and 4 th year. In this way, it was possible to know the profile of undergraduates and to analyze which domains are most impaired in the academic course.

KEYWORDS: Quality of life. Pharmacy students. University. 


\section{Introdução}

No começo da década de 90, cerca de um milhão e meio de pessoas estavam matriculadas no ensino superior no Brasil. Em 2011, o número aumentou para cerca de 6 milhões. Essas mudanças foram acompanhadas pelas políticas públicas de acesso ao ensino superior e os seus principais dobramentos para a inclusão profissional da população brasileira. No entanto, a discussão deste tema não envolve apenas os estudos das políticas públicas, mas também os resultados que elas promovem na formação profissional e o desenvolvimento humano dos estudantes universitários ${ }^{1}$.

Estudos recentes identificaram diminuição nos escores de Qualidade de Vida (QV) de acadêmicos de Jornalismo ${ }^{2}$, Psicologia $^{3}$, Enfermagem $^{4,5}$, Fisioterapia ${ }^{6}$ e Medicina $^{7,8}$. No curso de Farmácia, os três primeiros anos curriculares apresentam os maiores índices de estresse e redução dos escores de Qualidade de Vida Relacionada à Saúde (QVRS) mental ${ }^{9,10,11}$.

Definida pela Organização Mundial de Saúde ${ }^{12}$ como a percepção do indivíduo de sua posição na vida no contexto da cultura e sistema de valores nos quais ele vive e em relação aos seus objetivos, expectativas, padrões e preocupações, a QV é uma temática importante dentro do universo acadêmico, pois compreende um período de mudanças psicossociais influenciadas pelo ambiente altamente competitivo, noites sem dormir antes de provas e também pela ingestão de alimentos não saudáveis ${ }^{13}$.

Nesse sentido, o objetivo deste estudo foi de investigar a qualidade de vida dos graduandos de Farmácia da Universidade Federal do Oeste do Pará (UFOPA).

\section{Método}

Trata-se de um estudo prospectivo de corte transversal que foi realizado de maio a outubro de 2016, nos campus da UFOPA de Santarém. A pesquisa foi aprovada pelo Comitê de Ética em pesquisa da Universidade do Estado do Pará (CEP-UEPA) sob o número de parecer 1.447.073 e obteve financiamento da Fundação Amazônia de Amparo a Estudos e Pesquisas do Pará (FAPESPA) através do pagamento de bolsa de Iniciação Científica.

\section{Seleção e descrição dos participantes}

Os sujeitos inseridos na população deste estudo cumpriram os seguintes critérios de inclusão: (a) estar regularmente matriculado no curso de Farmácia da UFOPA; (b) ter 18 ou mais anos de idade; (c) assinar o Termo de Consentimento Livre e Esclarecido (TCLE). Foram excluídos os acadêmicos que não preencheram os pré-requisitos de inclusão.

O TCLE foi elaborado de acordo com a orientação da Resolução 510/2016 do Conselho Nacional de Saúde $(\mathrm{CNS})^{14}$, garantindo os princípios da bioética (beneficência, não maleficência, justiça e respeito à autonomia) ao longo de todos os passos deste estudo, assim como a garantia da confidencialidade das informações trazidas pelos participantes.

O Bacharelado em Farmácia da UFOPA tem o percurso acadêmico de 4 anos. Anualmente são ofertadas 40 vagas para a entrada de novos alunos. No período da coleta de dados, havia um total de 119 alunos matriculados. A amostra foi calculada através da fórmula ${ }^{15}: \mathrm{n}=\mathrm{o} 2$.p.q.N/E2.(N-1) + o2.p.q, na qual $\mathrm{n}$ corresponde ao tamanho da amostra; o2 ao nível de confiança escolhido em número de desvios $(68,3 \%=1$ desvio; $95,4 \%=2$ desvio; $99,7 \%=3$ desvio); $p$ à porcentagem das características pesquisadas na população; q à porcentagem do universo que não possui a característica pesquisada ( $q=100-p)$; $\mathrm{N}$ ao tamanho da população; e E2 ao erro de estimação permitido. Foi utilizado o erro de 5\%, nível de confiança com desvio 2.

A amostra $(n=92)$ foi distribuída em quatro grupos distintos $(n=23$ para cada grupo), de acordo com o ano de graduação. No entanto, o primeiro ano de graduação 
tinha apenas 13 alunos regularmente matriculados. Assim, o total amostral incluiu 82 participantes (Figura 1).

\section{Informação técnica}

Os instrumentos utilizados na obtenção dos dados desta pesquisa foram: ficha de dados sóciodemográficos (gênero, idade, estado civil, religião, ano de graduação e experiência de estágio), instrumento de avaliação da qualidade de vida da Organização Mundial de Saúde abreviado (WHOQOL-BREF) ${ }^{16}$ e questionário Medical Outcomes Study 36-item Short-Form Health Survey (SF-36) ${ }^{17}$.

OWHOQOL-BREF ${ }^{16}$ investigou a qualidade de vida geral, satisfação com a saúde, o domínio físico, o domínio psicológico, as relações sociais e o meio ambiente dos participantes. As respostas seguem uma escala de Likert de 1 a 5, quanto maior a pontuação melhor a qualidade de vida obedecendo a classificação: necessita melhorar (quando for 1 até 2,9); regular (3 até 3,9); boa (4 até 4,9) e muito boa (5). O SF-36 ${ }^{17}$ avaliou a capacidade funcional, aspectos físicos, dor, estado geral da saúde, vitalidade, aspectos sociais, aspectos emocionais e saúde mental. Os domínios são avaliados em uma escala que varia de 0 (zero) a 100 (cem), onde $0=$ pior e $100=$ melhor.

Todos os instrumentos utilizados no processo metodológico foram respondidos pelos graduandos de forma voluntária, após a assinatura do TCLE. Etapa realizada com uma turma de cada vez.

\section{Estatísticas}

Os resultados foram tabelados em software Microsoft Excel ${ }^{\circledR}$ 2010. As análises estatísticas foram realizadas no Graphpad Prism 6.0. Os dados foram descritos apresentando-se a frequência absoluta, relativa, medida de tendência central (média aritmética) e medida de dispersão (desvio padrão). Os valores não-paramétricos foram avaliados através do teste Kruskal-Wallis. Aplicou-se o teste ANOVA de um critério para os dados paramétricos.

\section{Resultados}

Dos 82 participantes, todos relataram experiência de estágio. O perfil sóciodemográfico dos estudantes de Farmácia da UFOPA apresentou predomínio de gênero feminino $(69,5 \%)$; solteiros $(90,24 \%)$ e de religião católica $(54,88 \%)$ (Tabela 1$)$. A média de idade não apresentou diferença estatisticamente significante entre os anos de graduação em Farmácia (Tabela 2).

$\mathrm{Na}$ Tabela 3, observam-se as informações acerca da qualidade de vida obtidas através do questionário WHOQOL-BREF, com respostas seguindo uma escala de Likert de 1 a 5 . Os menores escores da qualidade de vida geral, satisfação com a saúde, domínio físico e social foram observados no $1^{\circ}$ ano de graduação. Além disso, verificou-se que o $2^{\circ}$ ano apresenta os menores valores dos domínios psicológico e ambiente, quando comparado aos outros períodos do curso.

Em contrapartida, os maiores escores de qualidade de vida do WHOQOL-BREF foram obtidos no $3^{\circ}$ (domínios físico, psicológico e ambiente) e $4^{\circ}$ (qualidade de vida

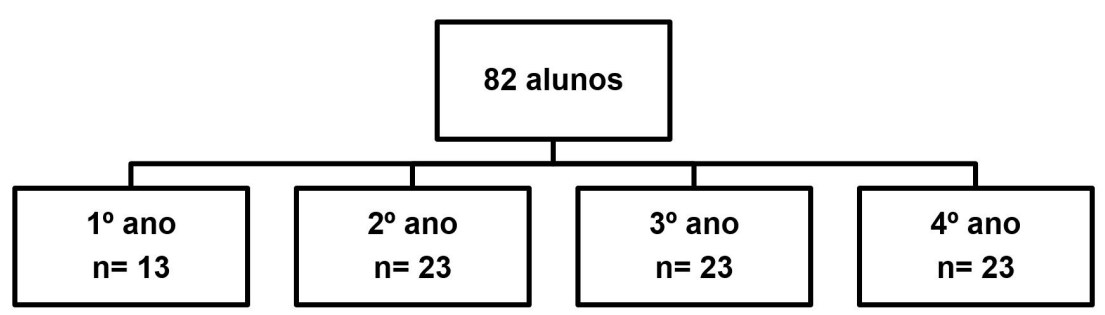

Figura 1. Distribuição dos grupos conforme o ano de graduação. 
Tabela 1. Distribuição dos acadêmicos do curso de Farmácia segundo dados sociodemográficos. Santarém, Pará, 2016.

\begin{tabular}{|c|c|c|c|c|c|c|}
\hline \multirow[b]{2}{*}{ VARIÁVEIS } & \multirow[b]{2}{*}{ Categorias } & \multicolumn{4}{|c|}{ CURSO: FARMÁCIA } & \multirow[b]{2}{*}{ Total n(\%) } \\
\hline & & $\begin{array}{l}4^{\circ} \text { ano } \\
(n=23)\end{array}$ & $\begin{array}{l}3^{\circ} \text { ano } \\
(\mathrm{n}=23)\end{array}$ & $\begin{array}{l}2^{\circ} \text { ano } \\
(\mathrm{n}=23)\end{array}$ & $\begin{array}{l}1^{\circ} \text { ano } \\
(\mathrm{n}=13)\end{array}$ & \\
\hline \multicolumn{7}{|l|}{ Sexo } \\
\hline & Masculino & 8 & 10 & 3 & 4 & $25(30,49 \%)$ \\
\hline & Feminino & 15 & 13 & 20 & 9 & $57(69,51 \%)$ \\
\hline \multicolumn{7}{|l|}{ Estado civil } \\
\hline & Solteiro & 19 & 23 & 20 & 12 & $74(90,24 \%)$ \\
\hline & Casado & 3 & - & 2 & 1 & $6(7,32 \%)$ \\
\hline & $\begin{array}{l}\text { União } \\
\text { Estável }\end{array}$ & 1 & - & 1 & - & $2(2,44 \%)$ \\
\hline \multicolumn{7}{|l|}{ Religião } \\
\hline & Evangélica & 7 & 9 & 5 & 3 & $24(29,27 \%)$ \\
\hline & Católica & 14 & 11 & 13 & 7 & $45(54,88 \%)$ \\
\hline & Adventista & 1 & 1 & - & - & $2(2,44 \%)$ \\
\hline & Agnóstico & - & 1 & - & - & $1(1,22 \%)$ \\
\hline & Budista & - & - & 1 & - & $1(1,22 \%)$ \\
\hline & $\begin{array}{l}\text { Testemunha } \\
\text { de Jeová }\end{array}$ & - & - & 1 & - & $1(1,22 \%)$ \\
\hline & Espírita & - & - & - & 1 & $1(1,22 \%)$ \\
\hline & Ateu & - & - & - & 1 & $1(1,22 \%)$ \\
\hline & Nenhuma & 1 & 1 & 3 & 1 & $6(7,32 \%)$ \\
\hline
\end{tabular}

Fonte: Autores (2017).

Tabela 2. Relação entre a idade e o ano de graduação.

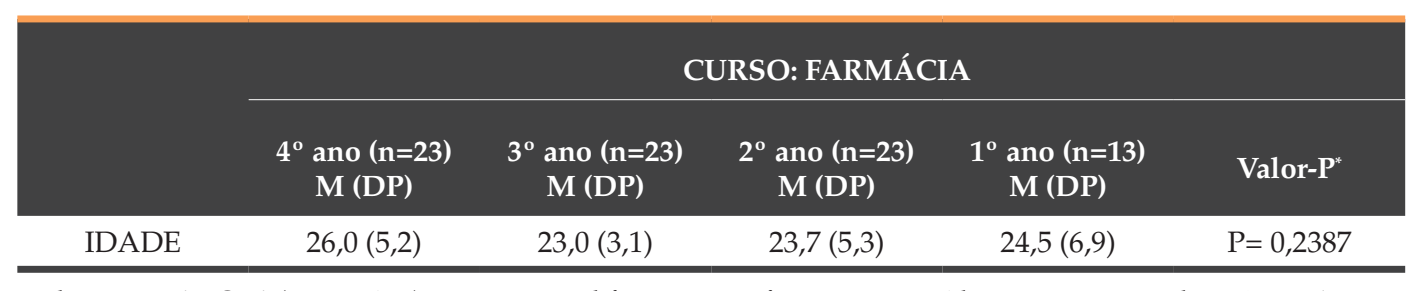

*Valor-P teste ANOVA (um critério); $\mathrm{P}<0,05$ para diferenças significativas. $\mathrm{M}=$ média; $\mathrm{DP}=$ Desvio Padrão. Fonte: Autores (2017).

geral, satisfação com a saúde, domínios social e ambiente) ano. No entanto, não foram encontrados valores estatisticamente significantes nos domínios avaliados entre os anos cursados.

Na Tabela 4, apresentam-se os dados obtidos através do questionário SF-36, onde cada domínio da QV recebeu um escore que variou de 0 a 100 . Neste instrumento, o $1^{\circ}$ ano apresentou o menor valor no domínio dos aspectos físicos e, em comparação com todos os outros anos, pode-se averiguar diferença estatisticamente significante $(p \leq 0,05)$. Além disso, $\mathrm{o} 1^{\circ}$ ano também evidenciou menores escores nos domínios da dor, aspectos sociais e emocionais. $\mathrm{O} 2^{\circ}$ ano apontou menores médias nos domínios capacidade funcional, estado geral de saúde, vitalidade e saúde mental. Porém, indicou o maior valor no aspecto emocional.

As maiores médias dos domínios capacidade funcional, aspectos físicos e sociais foram identificadas nos acadêmicos do $3^{\circ}$ ano. Já o $4^{\circ}$ ano, tem os maiores escores nos domínios da dor, estado geral da saúde, vitalidade e saúde mental. 
Tabela 3. Comparação entre a qualidade de vida e o ano cursado WHOQOL-BREF.

\begin{tabular}{|c|c|c|c|c|c|}
\hline \multirow{2}{*}{ DOMÍNIOS } & \multicolumn{5}{|c|}{ CURSO: FARMÁCIA } \\
\hline & $\begin{array}{c}4^{\circ} \text { ano }(\mathrm{n}=23) \\
M(\mathrm{DP})\end{array}$ & $\begin{array}{c}3^{\circ} \text { ano }(\mathrm{n}=23) \\
M(\mathrm{DP})\end{array}$ & $\begin{array}{c}2^{\circ} \text { ano }(n=23) \\
M(D P)\end{array}$ & $\begin{array}{c}1^{\circ} \text { ano }(\mathrm{n}=13) \\
M(\mathrm{DP})\end{array}$ & Valor-P ${ }^{*}$ \\
\hline $\begin{array}{l}\text { Qualidade de vida } \\
\text { geral }\end{array}$ & $3,57(0,79)$ & $3,48(0,79)$ & $3,57(0,79)$ & $3,38(0,77)$ & $\mathrm{P}=0,7280$ \\
\hline Satisfação com a saúde & $3,35(0,98)$ & $3,26(0,92)$ & $3,00(0,95)$ & $2,92(0,76)$ & $\mathrm{P}=0,2656$ \\
\hline Domínio Físico & $3,43(0,67)$ & $3,56(0,59)$ & $3,47(0,58)$ & $3,33(0,60)$ & $P=0,5670$ \\
\hline Domínio Psicológico & $3,62(0,60)$ & $3,68(0,66)$ & $3,26(0,85)$ & $3,31(0,62)$ & $\mathrm{P}=0,1583$ \\
\hline Domínio Social & $3,97(0,70)$ & $3,48(1,00)$ & $3,32(0,90)$ & $3,20(1,07)$ & $\mathrm{P}=0,0576$ \\
\hline Domínio Ambiente & $3,05(0,44)$ & $3,05(0,59)$ & $2,96(0,51)$ & $2,99(0,46)$ & $\mathrm{P}=0,9700$ \\
\hline
\end{tabular}

*Valor-P teste de Kruskal-Wallis; $\mathrm{P}<0.05$ para diferenças significativas. M= média; DP= Desvio Padrão. Fonte: Autores (2017).

Tabela 4. Comparação entre a qualidade de vida e o ano cursado SF-36.

\begin{tabular}{|c|c|c|c|c|c|}
\hline \multirow{2}{*}{ DOMÍNIOS } & \multicolumn{5}{|c|}{ CURSO: FARMÁCIA } \\
\hline & $\begin{array}{c}4^{\circ} \text { ano }(n=23) \\
M(D P)\end{array}$ & $\begin{array}{c}3^{\circ} \text { ano }(\mathrm{n}=23) \\
\mathrm{M}(\mathrm{DP})\end{array}$ & $\begin{array}{c}2^{\circ} \text { ano }(n=23) \\
M(D P)\end{array}$ & $\begin{array}{c}1^{\circ} \text { ano }(n=13) \\
M(D P)\end{array}$ & Valor-P* \\
\hline Capacidade Funcional & $86,74(18,19)$ & $90,65(11,11)$ & $84,13(11,14)$ & $84,23(14,27)$ & $\mathrm{P}=0,1652$ \\
\hline Aspectos Físicos & $47,83(33,64)$ & $71,74(34,79)$ & $63,04(43,22)$ & $38,46(34,78)$ & $\mathrm{P}=0,0343^{*}$ \\
\hline Dor & $67,91(27,25)$ & $63,87(17,97)$ & $59,61(20,17)$ & $58,00(26,01)$ & $\mathrm{P}=0,4952$ \\
\hline Estado Geral de Saúde & $57,78(17,14)$ & $51,65(13,04)$ & $48,35(18,48)$ & $56,31(14,68)$ & $P=0,2948$ \\
\hline Vitalidade & $54,78(17,48)$ & $50,22(17,22)$ & $40,00(18,15)$ & $43,85(22,00)$ & $\mathrm{P}=0,0558$ \\
\hline Aspectos Sociais & $63,04(25,42)$ & $67,39(26,58)$ & $59,24(26,17)$ & $55,35(28,11)$ & $\mathrm{P}=0,5324$ \\
\hline Aspecto Emocional & $57,97(41,71)$ & $44,92(37,08)$ & $59,42(37,55)$ & $41,02(41,18)$ & $\mathrm{P}=0,3822$ \\
\hline Saúde Mental & $67,13(17,48)$ & $63,65(18,72)$ & $54,96(22,21)$ & $55,69(19,22)$ & $\mathrm{P}=0,1740$ \\
\hline
\end{tabular}

*Valor-P teste de Kruskal-Wallis; P <0,05 para diferenças significativas. M= média; DP= Desvio Padrão. Fonte: Autores (2017).

\section{Discussão}

Sabe-se que o ingresso à universidade corresponde a um período onde os sujeitos estão expostos a diferentes influências psicossociais que podem desencadear situações estressoras, prejudicando a qualidade de vida dos estudantes ${ }^{2}$. A idade, o gênero, a condição socioeconômica, o aspecto subjetivo sobre felicidade e satisfação são alguns dos indicadores que estão inseridos na investigação da $\mathrm{QV}^{18}$. A presente amostra apresenta dados sóciodemográficos (gênero, a idade e o estado civil) semelhantes a outros estudos brasileiros ${ }^{19,20} \mathrm{com}$ acadêmicos do curso de Farmácia. Na variável idade, a maior média foi obtida pelo $4^{\circ}$ ano curricular seguida pelos demais anos, as médias variaram entre 23 a 26 anos de idade, dessa forma, corroborando com os resultados obtidos por outros autores ${ }^{9,10,11}$.

A hipótese específica de que há diferença nos escores de qualidade de vida dos instrumentos WHOQOL-BREF eSF-36 foi confirmada. Os dois questionários utilizados possuem diferenças metodológicas e não há na literatura outro estudo que apresente investigação da QV com ambos. Paro e colaboradores ${ }^{19}$, por exemplo, citam que as pesquisas de QV com universitários geralmente são abordadas através da aplicação do WHOQOL-BREF. Esta restrição, muitas vezes compromete a comparação com outros resultados sobre a mesma temática.

Os resultados do WHOQOL-BREF e do SF-36 apontam uma tendência de piores escores de QV no $1^{\circ}$ e $2^{\circ}$ ano curricular de Farmácia e os melhores escores de QV no $3^{\circ}$ e $4^{\circ}$ ano (Tabelas 3 e 4). Paro e colaboradores ${ }^{19}$, revelou situação semelhante 
obtendo piores escores de QV para os acadêmicos de Farmácia dos anos iniciais e melhores no $3^{\circ}$ e $4^{\circ}$ anos curriculares. Podendo ser justificado pelo fato de os alunos do $1^{\circ}$ e $2^{\circ}$ anos curriculares ainda não possuírem uma estratégia para os estudos, terem intensas disciplinas curriculares que fazem parte dos anos iniciais, refletindo assim, em uma dificuldade de adaptação à rotina universitária ${ }^{19}$.

Marshall e colaboradores ${ }^{11}$ identificaram que a intervenção familiar, finanças, relações pessoais, provas, testes didáticos, bem como tarefas fora da sala de aula são os principais motivos de estresse que influenciavam negativamente a QV dos acadêmicos do curso de Farmácia. Por outro lado, os escores de QV dos estudantes de Farmácia apresentam-se menos prejudicados quando comparados a outros cursos da área da saúde (enfermagem, fonoaudiologia e medicina), de forma que a QV nesses cursos apresentou-se mais comprometida no decorrer dos anos, devido às cargas de estágio e atividades profissionais serem distintas com relação ao curso de Farmácia ${ }^{19}$.

Entretanto, Oliveira ${ }^{20}$ pesquisou sobre a QV entre diversos cursos (Farmácia, Odontologia, Engenharia, Administração, Psicologia e Direito) e, utilizando o instrumento WHOQOL-BREF, verificou que o curso de Farmácia apresentou escore de QV baixo e as médias dos domínios físico e psicológico foram significativamente abaixo da média total dos estudantes. Com relação ao ano cursado, evidenciou que o ano parece não causar nenhum impacto com a QV dos graduandos ao iniciar e terminar o curso.

$\mathrm{Na}$ presente pesquisa, apenas o aspecto físico do SF-36 obteve diferença estatisticamente significante entre os anos curriculares de Farmácia ( $p$-valor $=0,0343$ ). Todavia, este resultado é diferente ao encontrado em outro achado de $Q V$, por meio do SF-36, o qual identificou que graduandos de Farmácia apresentam diferença estatisticamente significante apenas no aspecto vitalidade ( $1^{\circ}$ ano versus $4^{\circ}$ ano; $2^{\circ}$ ano versus $4^{\circ}$ ano; $p$-valor $\left.=0,041\right)^{19}$. Ambos os domínios, físico e vitalidade, estão relacionados com dedicação, esforço, força, disposição e dificuldade na realização de atividades ${ }^{17}$.

$\mathrm{O} 2^{\circ}$ ano aponta uma diminuição da QV relacionada à saúde mental no SF-36 e isto é corroborado pelo resultado no domínio psicológico do WHOQOL-BREF. Esses domínios estão relacionados com felicidade, nervosismo, sentimentos positivos e negativos, memória, autoestima, aparência, religião, segurança física, recursos financeiros, cuidados de saúde e sociais: qualidade, habilidades, lazer, poluição e transporte 16,17 .

Os melhores escores do domínio físico, tanto no WHOQOL-BREF quanto no SF-36, foram encontrados nos acadêmicos do $3^{\circ}$ ano. No $4^{\circ}$ ano, pode-se notar que a saúde e qualidade de vida geral estão melhores em relação aos outros anos. Oliveira ${ }^{5} \mathrm{em}$ seu estudo com graduandos do $8^{\circ}$ semestre do curso de enfermagem apresentou alguns fatores que auxiliam na QV como: situações de relaxamento; autoconfiança; laços de amizade; alimentação acessível; sono de qualidade entre outros.

\section{Conclusão}

O presente estudo analisou que os graduandos do curso de Farmácia da Universidade Federal do Oeste do Pará são predominantemente do gênero feminino, solteiros, católicos e com idade entre 18 a 45 anos. Entre os questionários aplicados podemos observar que os primeiros dois anos curriculares apresentam os piores escores de QV e os últimos dois anos curriculares têm os melhores escores tanto no instrumento WHOQOL-BREF quanto no SF-36. No entanto, apenas o SF-36 apresentou diferença estatisticamente significante no domínio do aspecto físico. Não sendo possível fazer uma relação se a idade influencia diretamente na qualidade de vida, pois, graduandos do $3^{\circ}$ ano curricular apresentam ótimos escores de $\mathrm{QV}$ e menor 
média de idade. Já os graduandos do $2^{\circ}$ ano, escores piores de QV e média de idade praticamente a mesma dos graduandos do $3^{\circ}$ ano.

Nesse sentido, os autores da pesquisa apontam a importância de realização de mais estudos com a aplicação de ambos os questionários de qualidade de vida (SF-36 e o WHOQOL-BREF) para melhor interpretação e discussão dos dados das amostras.

\section{Referências}

1. Brasil. Ministério da Educação. Conselho Nacional de Educação. Desenvolvimento, aprimoramento e consolidação de uma educação nacional de qualidade. Brasília; 2013. Projeto CNE/UNESCO 914BRZ1136.3.

2. Calais SL, Carrara K, Brum MM, Batista K, Yamada JK, Oliveira JRS. Stress entre calouros e veteranos de jornalismo. Estud Psicol. 2007;24(1):69-77. http://dx.doi.org/10.1590/S0103-166X2007000100008.

3. Costa CC, Bastiani M, Geyer JG, Calvetti PÜ, Muller MC, Moraes MLA. Qualidade de vida e bem-estar espiritual em universitários de Psicologia. Psicol Estud. 2008;13(2):249-55. http://dx.doi.org/10.1590/S1413-73722008000200007.

4. Furegato ARF, Santos JLF, Silva EC. Depressão entre estudantes de dois cursos de enfermagem: autoavaliação da saúde e fatores associados. Rev Bras Enferm. 2010;63(4):509-16. http://dx.doi.org/10.1590/S0034-71672010000400002.

5. Oliveira BM, Mininel VA, Felli VEA. Qualidade de vida de graduandos em enfermagem. Rev Bras Enferm. 2011;64(1):1305. http://dx.doi.org/10.1590/S0034-71672011000100019.

6. Bacchi CA, Candotti CT, Noll M, Minossi CES. Avaliação da qualidade de vida, da dor nas costas, da funcionalidade e de alterações da coluna vertebral de estudantes de fisioterapia. Motriz: Rev. Educ. Fis. 2013;19(2):243-51.

7. Figueiredo AM, Ribeiro GM, Reggiani ALM, et al. Percepções dos estudantes de medicina da Ufop sobre sua qualidade de vida. Rev Bras Educ Med. 2014;38(4):435-43. http://dx.doi.org/10.1590/S0100-55022014000400004.

8. Cardoso FAB Fo, Magalhães JF, Silva KML, Pereira ISSD. Perfil do Estudante de Medicina da Universidade do Estado do Rio Grande do Norte(UERN), 2013. Rev Bras Educ Med. 2015;39(1):32-40. http://dx.doi.org/10.1590/1981-52712015v39n1e01092014.

9. Payakachat N, Gubbins PO, Ragland D, Flowers SK, Stowe CD. Factors Associated With Health-Related Quality of Life of Student Pharmacists. Am J Pharm Educ. 2014;78(1):7. http://dx.doi.org/10.5688/ajpe7817.

10. Hirsch JD, Do AH, Hollenbach KA, Manoguerra AS, Adler DS. Students' health-related quality of life across the preclinical pharmacy curriculum. Am J Pharm Educ. 2009;73(8):147. http://dx.doi.org/10.5688/aj7308147.

11. Marshall LL, Allison A, Nykamp D, Lanke S. Perceived stress and quality of life among doctor of pharmacy students. Am J Pharm Educ. 2008;72(6):137. http://dx.doi.org/10.5688/aj7206137.

12. The Whoqol Group. The World Health Organization quality of life assessment (WHOQOL): position paper from the World Health Organization. Soc Sci Med. 1995;41(10):1403-9. http://dx.doi.org/10.1016/0277-9536(95)00112-K.

13. Nechita F, Streba CT, Vere CC, Nechita D, Rogoveanu I. Stress in Romanian first year nursing students. Curr Health Sci J. 2014;40(3):210-3. http://dx.doi.org/10.12865/CHSJ.40.03.10.

14. Brasil. Ministério da Saúde. Conselho Nacional de Saúde. Resolução 510/2016. Diário Oficial da União, Brasília, DF, 24 maio 2016.

15. Luchesa CJ, Chaves A No. Cálculo do tamanho da amostra nas pesquisas em administração. Curitiba: Edição do Autor; 2011. 43 p. [citado em 2017 Ago 14]. Disponível em: http://www.unicuritiba.edu.br/images/calculo_do_tamanho_da_amostra_-_ texto_final_para_impressapso1.pdf

16. Fleck MP, Louzada S, Xavier M, et al. Aplicação da versão em português do instrumento abreviado de avaliação da qualidade de vida WHOQOL-bref. Rev Saude Publica. 2000;34(2):178-83. http://dx.doi.org/10.1590/S0034-89102000000200012.

17. Ciconelli RM. Tradução para o português e validação do questionário genérico de avaliação de qualidade de vida "Medical Outcomes Study 36 - item Short-form Health Survey (SF-36)” [tese]. São Paulo: Universidade Federal de São Paulo; 1997 [citado em 2017 Ago 14]. Disponível em: http://repositorio.unifesp.br/handle/11600/15360

18. Pereira EF, Teixeira CS, Santos A. Qualidade de vida: abordagens, conceitos e avaliação. Rev Bras Educ Fís Esporte. 2012;26(2):241-50. http://dx.doi.org/10.1590/S1807-55092012000200007.

19. Paro CA, Bittencourt ZZLC. Qualidade de vida de graduandos da área da saúde. Rev Bras Educ Med. 2013;37(3):365-75. http://dx.doi.org/10.1590/S0100-55022013000300009.

20. Oliveira JAC. Qualidade de vida e desempenho acadêmico de graduandos [tese]. Campinas: Faculdade de Educação, Universidade Estadual de Campinas; 2006 [citado em 2017 Ago 14]. Disponível em: http://hdl.handle.net/123456789/1235

\section{Contribuição dos autores}

Luciana Fernandes Pastana Ramos e Juliana Érica Cirino Nascimento participaram do planejamento e execução do trabalho. 